\title{
A Tetris-like Model Showing a Universal Enhanced Flow Rate of a Hopper Discharging Hard Discs Through an Adjustable Inclusion
}

\author{
Guo-Jie Jason Gao ${ }^{1, *}$ \\ ${ }^{1}$ Department of Mathematical and Systems Engineering, \\ Shizuoka University, Hamamatsu, Shizuoka 432-8561, Japan
}

(Dated: September 28, 2018)

\begin{abstract}
In the literature, placing an inclusion near the orifice of a hopper, containing disc particles, has been experimentally and numerically reported to locally enhance the gravity-driven hopper flow rate. Moreover, the peaked flow rate can happen regardless of the interparticle friction, the inclusion geometry, or the disc dispersity. To reveal the fundamental reason causing this local effect, we propose a Tetris-like model that sequentially moves one disc particle at a time towards the hopper orifice. A Gaussian displacement function that independently controls a disc's movement in the horizontal or vertical direction, and the algorithm of the model accepts a movement as long as it creates no overlap between objects in the system. Our model creates an artificial steady probability-driven hopper flow without knowing the Newtonian dynamics which allows interparticle collaborative motion. Under specific conditions, we reproduce the enhanced flow rate and show that a moderate response time of the system and a flow rate difference between its value around the inclusion and its maximum without an inclusion are sufficient to explain this local effect with no Newton's laws involved.
\end{abstract}

\section{INTRODUCTION}

Multiple phases including gas, liquid and solid can coexist within short length scale comparable to particle size in nonequilibrium athermal systems such as a symmetric slot hopper continuously discharging granular particles under gravity. The empirical Beverloo equation captures the behavior of the funnel flow where only a part of particles near the center of the hopper flows, $Q \sim \rho \sqrt{g} W_{o}^{5 / 2}$, where $Q$ is the mass flow rate, $\rho$ is the bulk density of the granular medium, $g$ is gravity and $W_{o}$ is the orifice width of the hopper. On the other hand, the Johanson equation, $Q \sim \rho \sqrt{g} W_{o}^{3 / 2}$, describes the mass flow where almost all particles inside the hopper move simultaneously [1] 9.

Moreover, to globally increase the gravity-driven hopper flow rate, introducing more isotropic particles [9 15, increasing the particle dispersity [16 18], or placing an inclusion near the hopper orifice [19, 20] has been shown effective. The last strategy has also been shown experimentally and numerically to locally enhance the hopper flow rate of frictional particles recently, that is, the flow rate exhibits a local peak as an inclusion that creates a pressure reduction is placed at an optimal height above the hopper orifice 21 23]. However, this local effect cannot be explained by conventional continuum laws based on the assumption of constant density for hopper flows mentioned above.

In our recent study of frictionless particles interacting via the pairwise linear spring potential using molecular dynamics (MD) simulation, we also find the flow rate can be locally peaked and show that this local feature survives regardless of the interparticle friction, the particle

\footnotetext{
*koh.kokketsu@shizuoka.ac.jp, gjjgao@gmail.com
}

(a)

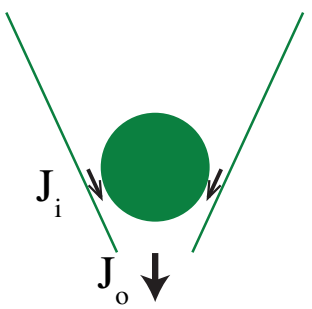

(b)

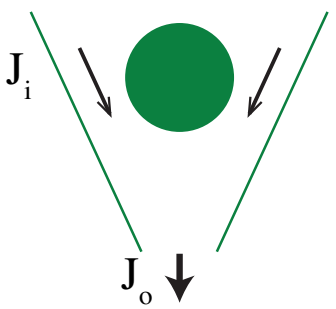

FIG. 1. (Color online) Schematic of a hopper (solid lines) containing an inclusion (circle). $J_{i}$ is the hopper flow rate at the inclusion height, with the part of the hopper lower than the center of the inclusion sliced off, and $J_{o}$ is the maximal flow rate while the hopper contains no inclusion. The hopper flow can be either (a) fluidized with $J_{i}<J_{o}$ or (b) clogging with $J_{i}>J_{o}$.

size dispersity and the inclusion geometry 24. The interparticle interactions do determine where the local flow rate happens. For example, a frictional system tends to exhibit its local flow rate peak with the inclusion placed higher than a frictionless system. In the light of our MD results, we have narrowed down possible reasons causing the locally enhanced flow rate to two candidates: 1) the interparticle collaborative motion due to the Newtonian dynamics and 2) the flow rate difference between its value $J_{i}$ at the inclusion height above the hopper orifice and its maximum $J_{o}$ while the hopper contains no inclusion, as shown schematically in Fig. 1, where (a) and (b) depict a fluidized flow regime with $J_{i}<J_{o}$ and a clogging flow regime with $J_{i}>J_{o}$, respectively. The local peak of the actual flow rate $J_{a}$ occurs because it can be boosted during the hopper flow transits from fluidized to clogging while $J_{i}$ becomes greater than $J_{o}$. To find out whether the Newtonian dynamics contributes indispensably to this local phenomenon, we propose a purely geometrical model that closely resembles the classical video 
game Tetris, where objects of various shapes fall down the playing field one at a time and can move left or right freely with a prescribed step size during the falling process without creating any overlap, except all objects are uniform circles in this study. A similar but more simplified Tetris-like model that contains rectangular objects occupying a 45-degree tilted square lattice and allows additional upward moving of objects had been used to study granular compaction under vibration 25. Our Tetris-like model produces an probability-driven hopper flow that can clog due to transient arching and particles blocking one another during position update with no Newtonian dynamics involved. Our results show that the local flow rate peak still exists under specific conditions, which serves as a decisive evidence that the Newtonian dynamics is not essential for this local phenomenon to happen.

Below we elaborate on our Tetris-like model, generating an artificial probability-driven hopper flow in section III followed by quantitative analysis of the flow rates with or without an inclusion in section model describing this local effect. We conclude our study in section IV

\section{NUMERICAL SIMULATION METHOD}

We develop a Tetris-like model to study the probability-driven hopper flow of monodisperse disc particles of diameter $d$, discharged from a geometrically symmetric hopper with a height $L=83 d$ and a hopper angle $\theta=0.4325$ radians, as shown schematically in Fig. 2(a). A circular inclusion of diameter $D=0.112 L$ is placed on the symmetric axis of the hopper and away from its orifice by a controllable distance $H$. The size ratio between the inclusion and a disc particle is $D / d=9.296$.

Within each position update in our Tetris-like model, each particle $i$ has exact one chance to change its horizontal $(\mathrm{x})$ and vertical $(\mathrm{y})$ positions from $\left(x_{i}^{\text {old }}, y_{i}^{\text {old }}\right)$ to $\left(x_{i}^{\text {new }}, y_{i}^{\text {new }}\right)$, based on

$$
x_{i}^{\text {new }}=x_{i}^{\text {old }}+N_{x}(0, \sigma)
$$

and

$$
y_{i}^{\text {new }}=y_{i}^{\text {old }}+\left|N_{y}(0, \alpha \sigma)\right|,
$$

where the two independent Gaussian functions, $N_{x}$ has a zero mean and a standard deviation $\sigma$, while $N_{y}$ also has a zero mean but a standard deviation $\alpha \sigma$. In both functions, $\sigma=0.05 d$, as shown exemplarily in Fig. 2(b). A larger value of $\alpha$, a variable in this study, represents a stronger trend of particles moving downwards to the orifice of the hopper, similar to discharging animate or inanimate particles through constrictions with increasing driving force [26]. We take the absolute value of $N_{y}$ so that particles move only towards the hopper orifice with no backward movement. The algorithm of the Tetrislike model accepts a change of position of particle $i$ if (a)

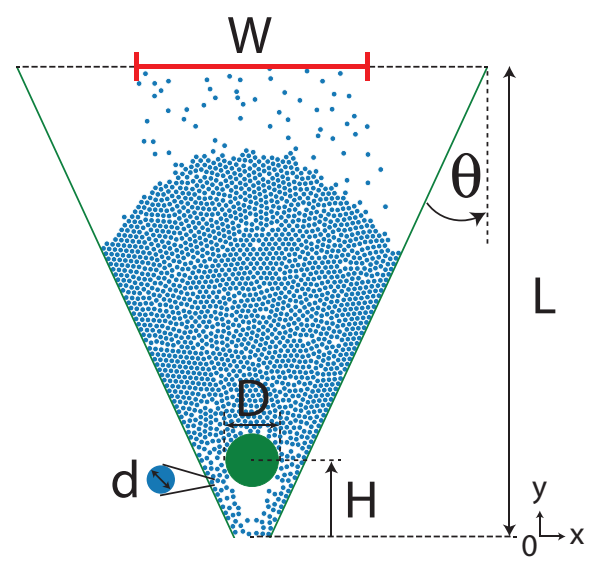

(b)

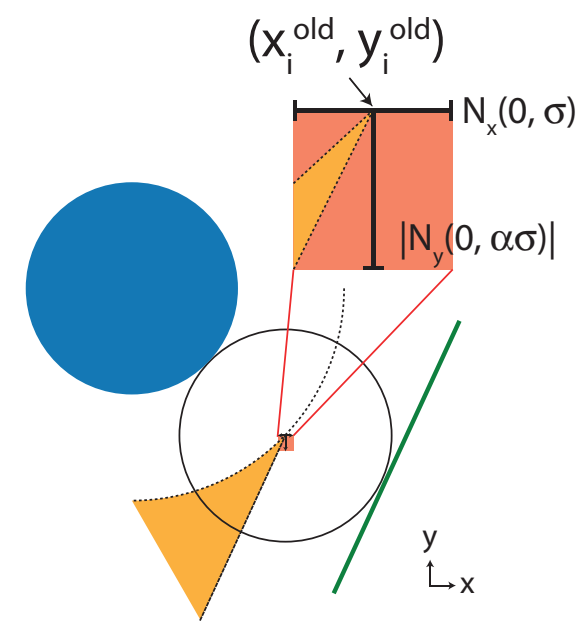

FIG. 2. (Color online) (a) The simulation setup of a symmetric hopper (slant solid lines) of equal height and top-width $L$ and hopper angle $\theta$. The hopper discharges disc particles (small circles) of diameter $d$ and possessing horizontal (x) and vertical (y) positions, which reenter the hopper from its top border, centered $(x=0)$ within $W=0.5 L$. An inclusion (large circle) of diameter $D$ sits at a height $H$ above the hopper orifice $(y=0)$. (b) The probabilistic Tetris-like model, exemplarily demonstrated by a particle (open circle) in between another particle (solid circle) and a wall (slant solid line). The fanned area (shaded) depicts a region where the sandwiched particle can move into at the next position update, governed by two independent Gaussian functions, $N_{x}$ and $N_{y}$ with zero means and standard deviations $\sigma$ and $\alpha \sigma$, respectively. $\sigma=0.05 d$ and $\alpha$ is a variable in this study.

it creates no overlap between the particle and any other objects in the system. Otherwise, the attempted position change will be discarded and particle $i$ stays unmoved. We update the positions of particles sequentially based on a random order that is different for each position update.

To measure the hopper flow rate $J_{a}$ while the inclusion is placed at a given height $H$ above the hopper orifice, we initiate a simulation with randomly arranged particles. There are $N=2048$ initially randomly placed particles in the system, which can fill the hopper up to about 


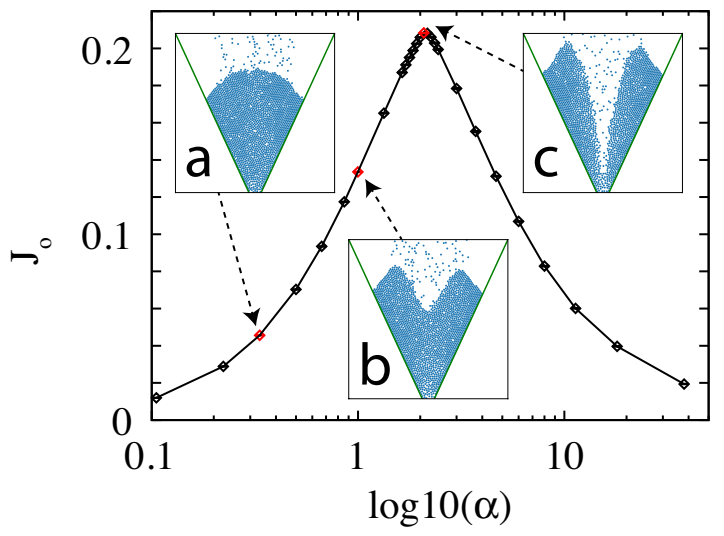

FIG. 3. (Color online) Hopper flow rate $J_{o}$ as a function of $\log _{10}(\alpha)$ while the hopper contains no inclusion. The error bars, smaller than the symbols in the plot, are obtained using 50 initial conditions. The insets show exemplary snapshots of $\alpha=$ (a) 1/3, (b) 1.0, and (c) 2.082 (diamonds), respectively.

$2 / 3$ of its height when the system reaches a steady state of a probability-driven hopper flow. To maintain a constant number of particles $N$, a particle dropping out of the hopper will reenter the system from the hopper's top border with its vertical (y) position artificially shifted by a distance $L$ and its horizontal (x) position reassigned randomly within $W \in[-L / 4, L / 4]$. The latter strategy is to maintain a steady hopper flow without particles piling up to the top border of the hopper, an undesirable boundary effect that affects the hopper flow rate. We then wait for 10,000 position updates so that the system becomes fully relaxed from the initial condition and reaches a steady probability-driven hopper flow. After that, we count the number of particles passing the hopper orifice within 990,000 position updates. For each value of $H$, we use 50 different initial conditions to calculate the average and the variance of the actual flow rate $J_{a}$ leaving the orifice in terms of number of particles leaving the hopper per update. We define $J_{o}$ as the value of $J_{a}$ while the hopper contains no inclusion. We also measure $J_{i}$, as the number of particles flowing through the internal passages between the inclusion and the hopper walls on its both sides per update, at the same height $H$ as the center of the inclusion is located above the orifice of the hopper in an identical way, except we put particles dropping below height $H$ back to the top of the hopper. This approach is essentially the same as measuring $J_{i}$ by slicing off the part of the hopper below the center of the inclusion so that the removed piece of hopper has no effect on $J_{i}$.

\section{RESULTS AND DISCUSSIONS}

To understand the behavior of the probability-driven hopper flow while the hopper contains no inclusion, we measured $J_{o}$ as a function of $\alpha$. The results are shown in
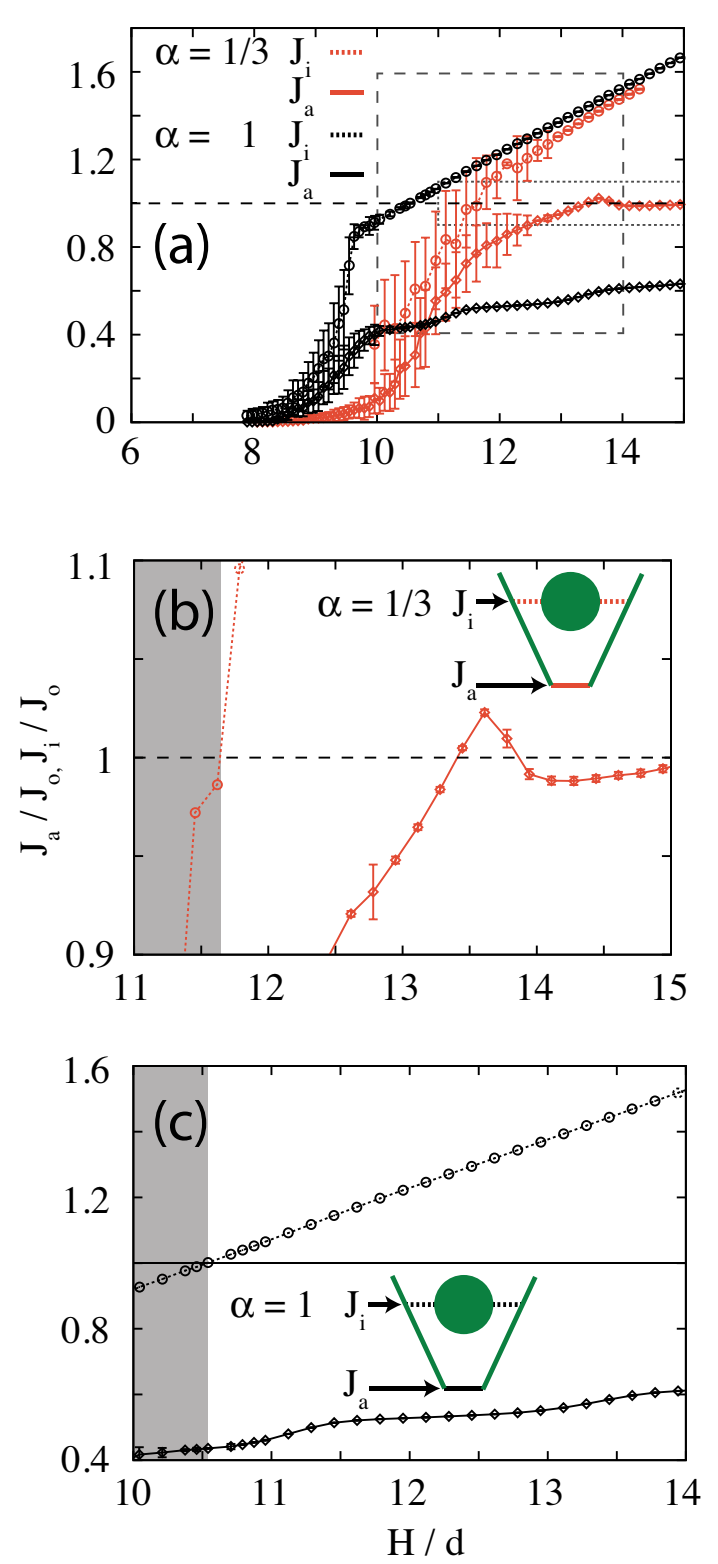

FIG. 4. (Color online) (a) Hopper flow rates $J_{i}$ measured at the inclusion height (dotted lines with circles) and $J_{a}$ measured at the hopper orifice (solid lines with diamonds) with $\alpha=1 / 3$ (the 2 nd and the 3rd curves from top) and 1.0 (the 1 st and the 4 th curves from top), respectively. The error bars, most of them smaller than the symbols in the plot, are obtained using 50 initial conditions. (b) and (c) show the zoomed plots of $J_{i}$ and $J_{a}$ in the dotted region of $\alpha=1 / 3$ and the dashed region of $\alpha=1.0$ in (a), separately. The fluidized flow regimes, where $J_{i}<J_{o}$ shown in Fig. 11(a), are shaded.

Fig. 3. $J_{o}$ initially increases monotonically with $\alpha$ until reaches its maximum at $\alpha \approx 2.082$. As can been seen in the insets of Fig. 3, in the monotonically increasing regime of $J_{o}$, the hopper flow performs a free surface like a bloated hump, a crater and a deeply-carved pit as $\alpha$ changes from $1 / 3,1.0$ to 2.082 , respectively. $J_{o}$ 


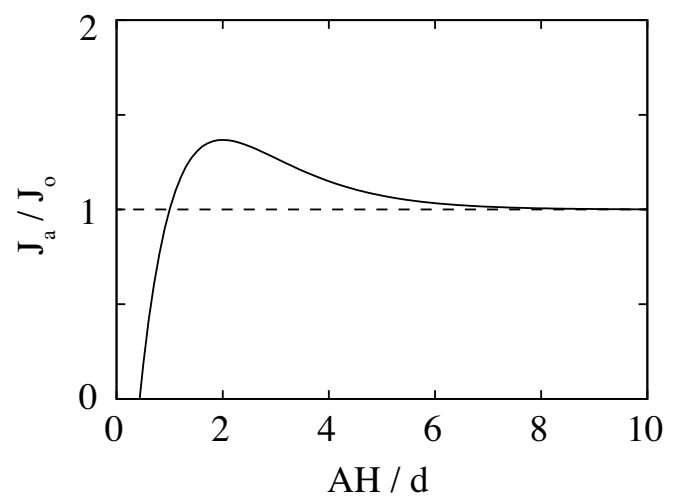

FIG. 5. Predicted $J_{a} / J_{o}$ of hopper flow, where $A H_{o} / d=1.0$ and $B / A=1.0$, described by the qualitative mathematical formula of Eqn. (3) with the same scaling factors $A$ and $B$.

then decreases to zero as $\alpha$ approaches infinity, where the rejection rate of relocating a particle becomes too high and a steady hopper flow is impossible. To avoid potential stagnant effect, we avoid this negatively-sloped regime of large $\alpha$ while studying the behavior of $J_{i}$ and $J_{a}$ when the hopper contains an inclusion.

Next, to verify our hypothesis that a flow rate difference between $J_{i}$ and $J_{o}$ may be sufficient to create a locally enhanced flow rate of $J_{a}$ in our Tetris-like model that creates a probability-driven hopper flow without the Newtonian dynamics, we measured $J_{i}$ and $J_{a}$, normalized by $J_{o}$, as a function of $H / d$. The results are shown in Fig. 4 (a). We chose $\alpha=1 / 3$ and 1.0, which give a low and a medium flow rates, respectively, in the positively sloped regime of $J_{o}(\alpha)$, as discussed in Fig. 3 above. We can see clearly in the zoomed Fig. 4(b) of $\alpha=1 / 3$ that soon after $J_{i}$ becomes higher than $J_{o}$ at $H / d \approx 11.6$, $J_{a}$ exhibits a local peak at $H / d \approx 13.6$. The local peak can be explained by a flow rate difference between $J_{i}$ at the inclusion height $H$ and $J_{o}$, together with an overall slower response time of the system, due to a smaller $\alpha$ and therefore a lower flow rate. The amount of particles passing the internal passages between the inclusion and the hopper walls while $J_{i}$ is moderately higher than $J_{o}$ is able to reach the hopper orifice before the system can response to it and limit $J_{a}$ to be no greater than $J_{o}$. As a result, $J_{a}>J_{o}$ is possible. As we lift the inclusion further away from the orifice, a flow of rate higher than $J_{o}$ has to travel longer to reach the hopper orifice and therefore has a higher chance to clog. The net result of this competition is a disappearing enhanced effect and eventually the value of $J_{a}$ saturates at $J_{o}$ as the inclusion sits too high to affect the flow rate $J_{a}$. On the other hand, although there still exists a greater than unity $J_{i} / J_{o}$ after $H / d>10.5$ in the zoomed Fig. 4(c) of a larger $\alpha=1.0$, we do not observe the enhanced effect. In this case, as $J_{i}$ become higher than $J_{o}$, the system always responses fast enough to effectively limit the actual flow rate $J_{a}$ out of the orifice below $J_{o}$.

Finally, we propose a simplified mathematical formula that qualitatively captures the flow mechanism described above. With a fixed value of $\alpha$, the flow rate $J_{a}$ leaving the hopper should be proportional to the strength of $J_{i} \sim A\left(H-H_{0}\right) / d$ at the inclusion height, where $A$ is a scaling factor, and $H_{0}$ is the height of the inclusion when particles in the hopper start to flow. $J_{i}$ grows linearly as a function of $H / d$ based on our simulation results, as shown in Fig. 4(b) and (c). As $J_{i}$ increases with the inclusion placed at a higher $H$, more and more particles can pass the two passages between the inclusion and the hopper walls, and the probability $P_{c}$ of particle clogging below the inclusion grows as well. We assume $P_{c}=e^{(B / A) J_{i}}$, where $B$ is another scaling factor, and $J_{a}$ is inversely proportional to $P_{c}$. Together, we obtain $J_{a} \sim J_{i} / P_{c}$, which can be explicitly expressed as

$$
J_{a}=J_{o}\left[1+\frac{A\left(H-H_{0}\right) / d}{e^{(B / A)\left(A\left(H-H_{0}\right) / d\right)}}\right] .
$$

A term of 1 is included so that $J_{a}$ approaches $J_{o}$ as the inclusion is placed at an $H$ far away from the hopper orifice and has no effect on $J_{a}$. Fig. 5 shows an exemplary hopper flow rate described by this formula. The actual forms of the linear relation, $J_{i} \sim A\left(H-H_{0}\right) / d$, and the assumed exponential term, $P_{c}=e^{(B / A) J_{i}}$, in Eqn. (3), however, are to be investigated in our next study.

\section{CONCLUSIONS}

In principle, the interparticle friction, the cooperative motion between particles and the geometry of grains and inclusion play important roles in granular hopper flow. For example, they determine precisely where the local peak of the hopper flow rate occurs, as previously reported in a gravity-driven frictional system, where disc particles passing through a round inclusion. Our previous study of frictionless particles using MD simulations has shown that altering the interparticle friction, the particle dispersity or the inclusion geometry affects the position of the local flow rate peak but does not necessarily eliminate it 24]. To investigate the importance of the long-range interparticle cooperative motion due to the Newtonian dynamics, we use a purely geometrical Tetris-like model that produces a probability-driven hopper flow and completely switch off the Newtonian interaction. Surprisingly, we can successfully reproduce the locally enhanced flow rate, and our results clearly show that a flow rate difference between its value $J_{i}$ at the inclusion height $H$ and its maximum $J_{o}$ while the hopper contains no inclusion, together with a slow response time of the system due to a moderate downward moving trend controlled by $\alpha$ in Eqn. (2), is sufficient to qualitatively reproduce this local phenomenon. As a result, the Newtonian dynamics is not indispensable for observing this local effect. This study serves as an example of deciphering a perplexing phenomenon in an athermal granular system by reducing its dynamics to the minimal to reveal the fundamental factor playing under the surface. 


\section{ACKNOWLEDGMENTS}

GJG gratefully acknowledges financial support from Shizuoka University startup funding. GJG also thanks Corey S. O'Hern for useful discussions.

[1] W. A. Beverloo, H. A. Leninger, and J. van de Valde, Chem. Eng. Sci. 15, 260 (1961).

[2] J. R. Johanson, Trans. Min. Engrs. AIME 232, 69 (1965).

[3] A. Janda, I. Zuriguel, and D. Maza, Phys. Rev. Lett. 108, 248001 (2012).

[4] H. M. Jaeger and S. R. Nagel, Science 255, 1523 (1992).

[5] K. Hutter and K. R. Rajagopal, Continuum Mech. Thermodyn. 6, 81 (1994).

[6] H. M. Jaeger, S. R. Nagel, and R. P. Behringer, Rev. Mod. Phys. 68, 1259 (1996).

[7] C. S. Campbell, Powder Technol. 162, 208 (2006).

[8] P. Jop, Y. Forterre, and O. Pouliquen, Nature 441, 727 (2006).

[9] C. C. Thomas and D. J. Durian, Phys. Rev. E 87, 052201 (2013).

[10] G. W. Baxter, R. P. Behringer, T. Fagert, and G. A. Johnson, Phys. Rev. Lett. 62, 2825 (1989).

[11] P. W. Cleary and M. L. Sawley, Appl. Math. Model. 26, 89 (2002).

[12] C. Wu and A. C. F. Cocks, Mechanics of Materials 38, 304 (2006).

[13] P. W. Cleary, Powder Technol. 179, 144 (2008).

[14] F. Y. Fraige, P. A. Langston, and G. Z. Chen, Powder Technol. 186, 224 (2008).
[15] D. Höhner, S. Wirtz, and V. Scherer, Powder Technol. 226, 16 (2012).

[16] A. V. Potapov and C. S. Campbell, Phys. Fluids 8, 2884 (1996).

[17] C. Denniston and H. Li, Phys. Rev. E 59, 3289 (1999).

[18] B. Remy, J. G. Khinast, and B. J. Glasser, Chem. Eng. Sci. 66, 1811 (2011).

[19] U. Tüzün and R. M. Nedderman, Chem. Eng. Sci. 40, 325 (1985).

[20] S. C. Yang and S. S. Hsiau, Powder Technol. 120, 244 (2001).

[21] I. Zuriguel, A. Janda, A. Garcimartín, C. Lozano, R. Arévalo, and D. Maza, Phys. Rev. Lett. 107, 278001 (2011).

[22] C. Lozano, A. Janda, A. Garcimartín, D. Maza, and I. Zuriguel, Phys. Rev. E 86, 031306 (2012).

[23] F. Alonso-Marroquin, S. I. Azeezullah, S. A. GalindoTorres, and L. M. Olsen-Kettle, Phys. Rev. E 85, 020301 (2012).

[24] G. J. Gao, J. Blawzdziewicz, and S. Ogata, arXiv:1807.05902 (2018).

[25] E. Caglioti, V. Loreto, H. J. Herrmann, and M. Nicodemi, Phys. Rev. Lett. 79, 1575 (1997).

[26] J. M. Pastor, A. Garcimartín, P. A. Gago, J. P. Peralta, C. Martín-Gómez, L. M. Ferrer, D. Maza, D. R. Parisi, L. A. Pugnaloni, and I. Zuriguel, Phys. Rev. E 92, 062817 (2015). 